INTERVENTIONAL CARDIOLOGY

\title{
OSA predicts cardiac risk in patients undergoing $\mathrm{PCl}$
}

Obstructive sleep apnoea (OSA) is an independent predictor of major adverse cardiac and cerebrovascular events in patients undergoing percutaneous coronary intervention (PCI). This finding was reported by investigators of the Sleep and Stent Study and published in Circulation.

OSA has been linked with increased risk of cardiovascular disease, but there are few studies on the prognostic effect of OSA in patients undergoing PCI. The Sleep and Stent study was a prospective, multicentre registry study designed to assess the association between OSA and cardiovascular outcomes in patients treated with PCI.

In total, 1,748 patients who underwent a successful PCI in at least one epicardial coronary artery were enrolled in the study. Participants completed the Berlin Questionnaire and the Epworth Sleepiness Scale questionnaire, and the apnoeahypopnoea index (AHI) - an index used to measure the severity of sleep apnoea - was calculated from sleep study tracings. The prespecified primary end point was a major adverse cardiac and cerebrovascular event (MACCE), a composite of cardiovascular mortality, nonfatal myocardial infarction, nonfatal stroke, and unplanned revascularization.

Overall, $80 \%$ of the patients were treated with drug-eluting stents and $6 \%$ with bioresorbable vascular scaffolds. OSA, defined as AHI $\geq 15$, was diagnosed in $45.3 \%$ of the patient cohort $(n=594)$. Patients with OSA were older, more likely to be male, had a higher BMI, and higher prevalence of hypertension and diabetes mellitus than those without OSA. During the median follow-up

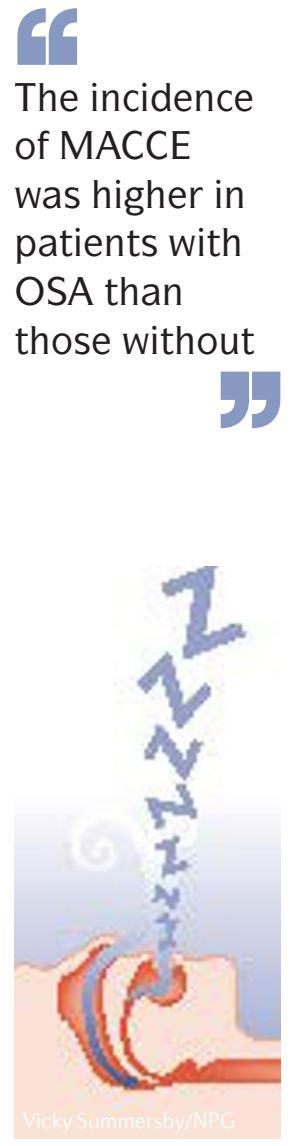

of 1.9 years, a MACCE occurred in 141 patients (3-year cumulative incidence estimate: $16.4 \%$ ), including cardiovascular death in 24 patients. The incidence of MACCE was higher in patients with OSA than in those without (3-year estimate: 18.9\% versus $14.0 \%$; $P=0.001)$. OSA was a predictor of MACCE independently of age, sex, BMI, diabetes, and hypertension (HR 1.57, 95\% CI 1.10-2.24, $P=0.013)$.

According to the study investigators, "the Sleep and Stent Study is the largest multinational collaborative effort examining OSA's effects on post-PCI cardiovascular outcomes to date". The investigators also emphasize the need for future studies to assess the effectiveness of OSA-directed therapies in reducing cardiovascular risk in these patients. Karina Huynh

ORIGINAL ARTICLE Lee, C.-H. et al. Obstructive sleep apnea and cardiovascular events after percutaneous coronary intervention. Circulation http://dx.doi.org/10.1161/CIRCULATIONAHA. 115.019392 (2016)

FURTHER READING Parati, G. et al. Heart failure and sleep disorders. Nat. Rev. Cardiol. http://dx.doi.org/10.1038/nrcardio.2016.71 (2016) 